\title{
ORIGINAL
}

\section{ASOCIACIÓN ENTRE EL GRADO DE CONTROL DE LA HIPERTENSIÓN ARTERIAL, LA COMORBILIDAD Y LOS COSTES EN PERSONAS DE MÁS DE 30 AÑOS DURANTE EL AÑO 2006 (*)}

\author{
Antoni Sicras-Mainar (1), Soledad Velasco-Velasco (1), Josep Ramón Llopart-López (1), Nuria \\ González-Rojas Guix (2), Chenco Clemente-Igeño (2) y Ruth Navarro-Artieda (3) \\ (1) Dirección de Planificación, Badalona Serveis Assistencials SA, Badalona, Barcelona. \\ (2) Boehringer-Ingelheim SCV, Barcelona. \\ (3) Servicio de Documentación, Hospital Germans Trias y Pujol, Badalona, Barcelona.
}

\section{RESUMEN}

Fundamento. La hipertensión arterial (HTA) es uno de los principales motivos de consulta de los centros de atención primaria (AP). El objetivo del estudio fue determinar la asociación entre el grado de control de la HTA, la comorbilidad y los costes directos en atención primaria.

Métodos. Diseño retrospectivo-multicéntrico. Se incluyó a sujetos mayores de 30 años pertenecientes a cinco equipos de AP (año 2006). Criterios: buen control $(<140 / 90$, y $<130 / 80$ $\mathrm{mmHg}$ en personas diabéticas y presencia de enfermedad cardiovascular $[\mathrm{ECV}])$. Principales medidas: generales, ECV, índice de Charlson, casuística/comorbilidad (Adjusted Clinical Groups), parámetros clínicos y costes directos (fijos/semifijos y variables [medicamentos, pruebas y derivaciones]). Análisis de regresión logística y de ANCOVA para la corrección del modelo, $\mathrm{p}<0,05$.

Resultados. La prevalencia de HTA fue del 26,5\% (edad media: 67,1 años; varones: 43,5\%). El buen control fue del $52,0 \%$ (IC: $51,2-52,8 \%$ ). El mal control tuvo una relación independiente con la diabetes $(\mathrm{OR}=3,8)$, el $\mathrm{ECV}(\mathrm{OR}=2,2)$ y los varones $(\mathrm{OR}=1,2), \mathrm{p}<0,001$. El promedio/unitario/año del coste directo corregido fue de $1.202,13$ vs. $1.183,55 €$ $(\mathrm{p}=0,032)$.

Conclusiones. Los pacientes en situación de mal control muestran una mayor carga de morbilidad y un similar coste sanitario.

Palabras clave: Hipertensión arterial. Morbilidad. Coste. Atención primaria de salud. Control.

Correspondencia:

Antoni Sicras Mainar

Dirección de Planificación y Desarrollo Organizativo

Calle Gaietà Soler, 6-8 entlo. 08911 Badalona. Barcelona.

Correo electrónico: asicras@bsa.cat

\section{ABSTRACT \\ Relationship among the Degree of Control of Arterial Hypertension, Comorbidity and Costs in Individuals over Age 30 during 2006}

Background. Arterial hypertension is one of the main reasons for primary care consultations. This study is aimed at determining the relationship among the degree to which arterial hypertension is controlled, comorbidity and the direct costs in primary care.

Methods. Retrospective, multi-centre design. Subjects over 30 years of age pertaining to five primary care teams (2006) were included. Criteria: good control $(<140 / 90$ and $<130 / 80 \mathrm{mmHg}$ in diabetics and those with cardiovascular disease [CVD]. Main general measurements, CVD, Charlson index, casuistic/comorbidity (Adjusted Clinical Groups), clinical parameters and direct costs (fixed/semifixed and variable costs) [medications, tests and referrals]) Logic regression and ANCOVA for correcting the model, $\mathrm{p}<0.05$.

Results: The prevalence of arterial hypertension was $26.5 \%$ (mean age: 67.1 years; males: $43.5 \%$ ). Good control totalled $52.0 \%$ (CI: $51.2-52.8 \%$ ). Poor control was independently related to diabetes (Odds Ratio $=3.8$ ), CVD (Odds Ratio $=2.2$ ) and males (Odds Ratio $=1.2$ ), $\mathrm{p}<0.001$. The average/direct unit cost/year was $1,202.13 €$ vs. $1,183.55 €(\mathrm{p}=0.032)$.

Conclusions. Those individuals whose arterial hypertension was poorly controlled displayed a greater burden of morbidity and a similar healthcare cost in comparison to those under good control.

Key words: Hypertension arterial. Morbidity. Cost. Primary healthcare.

(*) El trabajo fue parcialmente financiado por BoehringerIngelheim y el Fondo de Investigaciones Sanitarias de la Seguridad Social (PI 05/2837). 


\section{INTRODUCCIÓN}

El aumento de la esperanza de vida está causando que la hipertensión arterial (HTA) sea uno de los principales motivos de consulta de los centros de atención primaria (AP), constituyendo un importante problema de salud pública ${ }^{1}$. En la población general adulta española la prevalencia de la HTA es de aproximadamente un $35 \%$, llegando al $40 \%$ en edades medias y al $65 \%$ en las personas mayores de 60 años, y posiblemente seguirá aumentando, puesto que el progresivo envejecimiento poblacional, entre otros factores, podría repercutir elevando la prevalencia y el uso de recursos en los próximos años ${ }^{2-4}$.

En este aspecto, el grado de control de la presión arterial conseguido en algunos estudios españoles oscila entre el 35-40\%; mientras que el buen control en los sujetos diabéticos varía en niveles comprendidos entre el 7-10\%. Este grado de control es mejorable, pero mediante intervenciones específicas se van incrementando estos porcentajes paulatinamente en el tiempo ${ }^{2-8}$.

El nivel de control de la HTA de los pacientes es una medida importante porque se asocia con la comorbilidad y el consumo de recursos sanitarios. En relación con este punto es importante señalar que las evidencias disponibles en España y en otros países acerca de la asociación de estas tres variables son escasas ${ }^{1,9-10}$. Consecuentemente, los estudios dirigidos a mejorar el conocimiento sobre este tema resultan pertinentes.

El objetivo del estudio fue determinar la asociación entre el grado de control de la HTA, la comorbilidad y los costes directos en el ámbito de la AP de salud.

\section{MATERIAL Y METODOS}

Se realizó un estudio retrospectivo de carácter multicéntrico, realizado a partir de la revisión de los registros médicos de sujetos seguidos en régimen ambulatorio. La población de estudio estuvo formada por pacientes de cinco centros reformados de AP gestionados por Badalona Serveis Assistencials SA. Se incluyó en el estudio a todos los sujetos mayores de 30 años que demandaron asistencia durante todo el año 2006 (enero-diciembre). Fueron excluidos los sujetos trasladados a otros centros, los desplazados o fuera de zona y los atendidos por los especialistas integrados. También se excluyeron aquellos pacientes menores de 31 años, puesto que la prevalencia de HTA, diabetes y enfermedad cardiovascular es baja, y poco comparable sus resultados de buen control con la mayoría de los estudios disponibles. Se consideró buen control para cifras de tensión arterial $<140 / 90$ y $<130 / 80 \mathrm{mmHg}$ en personas diabéticas y con presencia de enfermedad cardiovascular (ECV), siguiendo los criterios de la $\mathrm{ESH} / \mathrm{ESC}^{11}$. El procedimiento de medida de la presión arterial es el siguiente: la medida basal se realiza en reposo psíquico y físico, dejando al paciente sentado/estirado durante varios minutos antes de iniciar la determinación (retirando las prendas de vestir del brazo). El manguito (diferentes tamaños), queda a la altura del corazón y se ajusta sin holgura ni compresión. En ancianos se realiza una toma en ortostatismo tras un minuto en bipedestación, y en jóvenes una medida en la pierna para excluir coartación. Para el diagnóstico de la hipertensión se realizan al menos dos medidas por visita (separación de 1-2 minutos) y en tres series en semanas diferentes (en ambos brazos para descartar una enfermedad vascular periférica). En todos los centros las mediciones se realizan mediante aparatos automáticos validados y calibrados en el último año; y los valores obtenidos para el estudio, proceden de la toma del médico o la enfermera en la propia consulta ${ }^{11}$. 
Las principales medidas fueron: generales (edad, sexo), presencia de ECV (angor péctoris, infarto agudo de miocardio, accidente vasculocerebral y arteriopatía periférica), índice de Charlson (severidad), casuística/comorbilidad general, parámetros clínicos (lípidos, glucemia, colesterol total y fracciones, etc.) y modelo de costes directos. Las diferentes comorbilidades se definieron como una enfermedad clínica específica obtenida a partir de la Clasificación Internacional de la AP (CIAP-2 $)^{12}$. Los valores obtenidos de tensión arterial y parámetros bioquímicos fueron obtenidos a partir de la última determinación durante el período de estudio. La carga de morbilidad general de cada paciente/año se cuantificó a partir de los Adjusted Clinical Groups. El algoritmo de funcionamiento del Grouper $\mathrm{ACG}^{\circledR}$ está constituido por una serie de pasos consecutivos hasta la obtención de los 106 ACG grupos mutuamente excluyentes para cada paciente atendido ${ }^{13}$. El aplicativo ACG proporciona los pesos relativos medios americanos (PRM) de cada grupo respecto al coste medio total y las bandas de utilización de recursos (BUR), con lo que cada paciente en función de su morbilidad queda agrupado en una de las 5 categorías mutuamente excluyentes (rango: 1-usuarios sanos a 5-morbilidad muy elevada).

Los recursos sanitarios obtenidos a partir de los registros propios de cada centro fueron las visitas o citas realizadas en el centro de AP, las derivaciones efectuadas a los especialistas de referencia, las solicitudes de las pruebas complementarias de soporte y la prescripción farmacéutica a cargo del CatSalut. El diseño del sistema de costes se definió teniendo en cuenta las características de la organización y el grado de desarrollo de los sistemas de información disponibles. La unidad de producto asistencial que sirvió de base al cálculo final fue el coste/paciente atendido durante el período de estudio. Según su dependencia con el volumen de actividad desarrollada se consideraron los costes fijos/semifijos (con criterio de impu- tación) y los costes variables. Se consideraron como costes fijos/semifijos (estructura) los relativos a personal (sueldos y salarios), bienes de consumo y un conjunto de gastos pertenecientes a los servicios externos, de acuerdo con el Plan General Contable para los Centros de Asistencia Sanitaria, y como variables, los relacionados con las solicitudes diagnósticas, terapéuticas o derivaciones efectuadas por los profesionales del centro. Los diferentes conceptos de estudio y su valoración económica fueron los siguientes: a) pruebas complementarias: incluye las de laboratorio (gasto medio/petición; 18,50€), radiología convencional (tarifa interna/prueba solicitada; rango: 7-120€) y pruebas de soporte (tarifa convencional/prueba solicitada; rango: 5-85€); b) derivaciones efectuadas a los especialistas de referencia o a los centros hospitalarios de carácter ordinario o urgentes (tarifa adaptada/derivación; 98€), y c) prescripciones (recetas médicas agudas, crónicas o a demanda; precio de venta al público/envase; fuente CatSalut). Las tarifas utilizadas procedieron de estudios de contabilidad analítica realizados en la propia organización o de precios establecidos por el CatSalut. A partir de los costes semifijos se obtuvo un coste medio por visita realizada (coste/visita: $20 €)$ y se efectuó un reparto directo final para cada paciente atendido durante el período de estudio. Por tanto, el coste por paciente $(\mathrm{Cp})$ fue: $\mathrm{Cp}=($ coste medio por visita $€$ número de visitas [costes semifijos]) + costes variables (laboratorio [gasto/petición] + radiología [tarifa/prueba] + pruebas de soportes [tarifa/prueba] + derivaciones [tarifa/derivación] + gasto farmacéutico [precio venta/envase] $)^{14}$.

Como paso previo al análisis se revisó cuidadosamente la calidad de los datos, respetándose la confidencialidad de los registros marcada por la ley. Se efectuó un análisis de regresión logística (ajuste: edad, sexo, índice de Charlson) con procedimiento enter, para la corrección del modelo. La comparación del coste ambulatorio se realizó según las recomendaciones de Thomp- 
son y Barber ${ }^{15}$ mediante el análisis de la covarianza (ANCOVA), con el sexo, la edad y las BUR como covariables (procedimiento: estimación de medias marginales; ajuste de Bonferroni). Se utilizó el programa SPSSWIN versión 12, estableciendo una significación estadística para valores de $\mathrm{p}<0,05$.

\section{RESULTADOS}

De 57.025 sujetos mayores de 30 años, un $26,5 \%(n=13.636$; intervalos de confianza [IC], 25,8-27,2\%) presentó HTA (edad media: 67,1 $\pm 12,3$ años; varones: 43,5\%; morbilidad moderada: $73,8 \%$, y coste total: 16,4 millones/euros. El buen

Tabla 1

Distribución de las variables generales, eventos cardiovasculares y comorbilidades según el grado control de la hipertensión

\begin{tabular}{|c|c|c|c|c|c|c|}
\hline \multirow{2}{*}{$\begin{array}{l}\text { Variables de estudio } \\
\text { Sujetos, \% }\end{array}$} & \multirow{2}{*}{$\begin{array}{c}\text { Mal control } \\
\mathrm{n}=6.545(48,0 \%)\end{array}$} & \multirow{2}{*}{$\begin{array}{c}\text { Bueno control } \\
n=7.091(52,0 \%)\end{array}$} & \multirow{2}{*}{$\begin{array}{c}\text { Total } \\
\mathbf{n}=13.636(100,0 \%)\end{array}$} & \multirow{2}{*}{$\mathbf{p}$} & \multicolumn{2}{|c|}{ Modelo corregido $^{(1)}$} \\
\hline & & & & & OR & IC del $95 \%$ \\
\hline $\begin{array}{l}\text { Enfermedad cardiovascular Generales } \\
\text { Edad media años } \\
\text { Rangos: } \\
\text { 31-44 años } \\
\text { 35-64 años } \\
\text { 65-74 años } \\
\text { > } 74 \text { años } \\
\text { Género (varones) } \\
\text { Promedio de índice de Charlson } \\
\text { Promedio de visitas/año }\end{array}$ & $\begin{array}{c}25,7 \% \\
67,9 \pm 12,1 \\
3,6 \% \\
34,4 \% \\
28,7 \% \\
33,2 \% \\
46,2 \% \\
0,7 \pm 0,7 \\
13,7 \pm 11,0\end{array}$ & $\begin{array}{c}12,8 \% \\
66,3 \pm 12,4 \\
4,6 \% \\
39,9 \% \\
27,2 \% \\
28,3 \% \\
40,9 \% \\
0,5 \pm 0,7 \\
13,0 \pm 10,5\end{array}$ & $\begin{array}{c}19,0 \% \\
67,1 \pm 12,3 \\
4,1 \% \\
37,3 \% \\
28,0 \% \\
30,7 \% \\
43,5 \% \\
0,6 \pm 0,7 \\
13,4 \pm 10,7\end{array}$ & $\begin{array}{l}<0,001 \\
<0,001 \\
<0,001 \\
<0,001 \\
<0,001 \\
0,041\end{array}$ & $\begin{array}{l}2,0 \\
-\end{array}$ & $1,1-1,3$ \\
\hline $\begin{array}{l}\text { Bandas de utilización de recursos (BUR) } \\
\text { 1: morbilidad muy baja } \\
\text { 2: morbilidad baja } \\
\text { 3: morbilidad moderada } \\
\text { 4: morbilidad elevada } \\
\text { 5: morbilidad muy elevada } \\
\text { Pesos relativos medios americanos (PRM) }\end{array}$ & $\begin{array}{r}2,1 \% \\
12,4 \% \\
73,1 \% \\
10,8 \% \\
1,6 \% \\
2,7 \pm 2,7\end{array}$ & $\begin{array}{c}2,4 \% \\
12,1 \% \\
74,4 \% \\
10,0 \% \\
1,1 \% \\
2,5 \pm 2,4\end{array}$ & $\begin{array}{c}2,2 \% \\
12,2 \% \\
73,8 \% \\
10,4 \% \\
1,3 \% \\
2,6 \pm 2,6\end{array}$ & $\begin{array}{l}0,038 \\
0,041\end{array}$ & & \\
\hline $\begin{array}{l}\text { Comorbilidades asociadas } \\
\text { Diabetes mellitus } \\
\text { Dislipemia } \\
\text { Obesidad } \\
\text { Fumadores } \\
\text { Alcoholismo }\end{array}$ & $\begin{array}{r}38,4 \% \\
49,6 \% \\
22,3 \% \\
16,5 \% \\
2,7 \%\end{array}$ & $\begin{array}{r}13,4 \% \\
44,6 \% \\
21,6 \% \\
15,2 \% \\
2,1 \%\end{array}$ & $\begin{array}{r}25,4 \% \\
47,0 \% \\
22,0 \% \\
15,8 \% \\
2,4 \%\end{array}$ & $\begin{array}{c}<0,001 \\
<0,001 \\
\text { NS } \\
0,028 \\
0,016\end{array}$ & $\begin{array}{l}3,8 \\
- \\
- \\
-\end{array}$ & $1,8-2,2$ \\
\hline
\end{tabular}

Valores expresados en porcentaje o media \pm desviación estándar; p: significación estadística; NS: no significativo; IC: intervalos de confianza del 95\%. (1) Modelo logístico: significación estadística, p<0,001; OR: odds ratio; modelo final corregido por edad, género e índice de Charlson (procedimiento: enter; estadístico: Wald).

Tabla 2

Parámetros clínicos y modelo de costes directos brutos según el grado control de la hipertensión

\begin{tabular}{|c|c|c|c|c|}
\hline $\begin{array}{c}\text { Variables de estudio } \\
\text { Sujetos, \% }\end{array}$ & $\begin{array}{c}\text { Mal control } \\
\mathrm{n}=6.545(\mathbf{4 8 , 0 \%})\end{array}$ & $\begin{array}{c}\text { Bueno control } \\
n=7.091(52,0 \%)\end{array}$ & $\begin{array}{c}\text { Total } \\
\mathrm{n}=13.636 \\
(\mathbf{1 0 0 , 0 \%})\end{array}$ & $\mathbf{p}$ \\
\hline $\begin{array}{l}\text { Parámetros clínicos } \\
\text { Índice de masa corporal, } \mathrm{kg} / \mathrm{m}^{2} \\
\text { Glicemia basal, } \mathrm{mg} / \mathrm{dl} \\
\text { Triglicéridos séricos, } \mathrm{mg} / \mathrm{dl} \\
\text { Colesterol total, } \mathrm{mg} / \mathrm{dl} \\
\text { cHDL-colesterol, } \mathrm{mg} / \mathrm{dl} \\
\text { cLDL-colesterol, } \mathrm{mg} / \mathrm{dl} \\
\text { Índice de riesgo cardiovascular }\end{array}$ & $\begin{array}{c}30,5 \pm 5,0 \\
114,7 \pm 36,9 \\
140,6 \pm 88,0 \\
203,2 \pm 40,6 \\
56,4 \pm 17,1 \\
119,9 \pm 35,8 \\
18,6 \pm 10,01\end{array}$ & $\begin{array}{c}29,7 \pm 4,9 \\
102,1 \pm 26,6 \\
129,8 \pm 84,6 \\
207,3 \pm 38,4 \\
58,1 \pm 17,3 \\
124,5 \pm 35,4 \\
14,5 \pm 9,3\end{array}$ & $\begin{array}{c}30,1 \pm 5,0 \\
108,1 \pm 32,6 \\
134,9 \pm 86,4 \\
205,6 \pm 39,5 \\
57,3 \pm 17,2 \\
122,3 \pm 35,6 \\
16,4 \pm 9,9\end{array}$ & $\begin{array}{l}<0,001 \\
<0,001 \\
<0,001 \\
<0,001 \\
<0,001 \\
<0,001 \\
<0,001\end{array}$ \\
\hline $\begin{array}{l}\text { Modelo de costes brutos } \\
\text { Coste fijo/semifijo } \\
\text { Coste variable } \\
\text { - Derivaciones a especialistas } \\
\text { - Prescripción farmacéutica } \\
\text { Coste total en Atención Primaria }\end{array}$ & $\begin{array}{c}249,64 \pm 199,54 \\
1.028,57 \pm 977,05 \\
100,18 \pm 135,02 \\
896,70 \pm 933,82 \\
1.278,2 \pm 1.082,86\end{array}$ & $\begin{array}{c}237,10 \pm 191,36 \\
893,55 \pm 901,27 \\
96,89 \pm 126,41 \\
767,68 \pm 865,59 \\
1.130,65 \pm 1.003,23\end{array}$ & $\begin{array}{c}243,13 \pm 195,42 \\
958,36 \pm 940,80 \\
98,47 \pm 130,62 \\
829,61 \pm 901,26 \\
1.201,49 \pm 1.041,78\end{array}$ & $\begin{array}{l}<0,001 \\
<0,001 \\
0,043 \\
<0,001 \\
<0,001\end{array}$ \\
\hline
\end{tabular}

Valores expresados en porcentaje o media \pm desviación estándar; p: significación estadística. 
Tabla 3

Modelo de costes directos corregidos (ANCOVA) según el grado control de la hipertensión

\begin{tabular}{|c|c|c|c|c|c|}
\hline \multirow{2}{*}{$\begin{array}{c}\text { Variables de estudio } \\
\text { Sujetos, } \% \\
\text { Coste unitario total corregio }{ }^{(1)}\end{array}$} & \multicolumn{2}{|c|}{$\begin{array}{c}\text { Mal control } \\
n=6.545(48,0 \%)\end{array}$} & \multicolumn{2}{|c|}{$\begin{array}{c}\text { Bueno control } \\
\mathrm{n}=7.091(52,0 \%)\end{array}$} & \multirow{2}{*}{$\mathbf{p}$} \\
\hline & Promedio & IC del $95 \%$ & Promedio & IC del $95 \%$ & \\
\hline Coste fijo/semifijo & 242,73 & $238,35-247,11$ & 238,70 & $234,20-243,19$ & 0,033 \\
\hline Coste variable & 963,80 & $942,89-984,70$ & 940,83 & $920,49-961,17$ & 0,029 \\
\hline - Derivaciones a especialistas & 98,78 & $95,71-101,85$ & 96,71 & $93,55-99,87$ & NS \\
\hline - Prescripción farmarmacéutica & 836,22 & $816,14-856,31$ & 813,81 & $816,14-856,31$ & 0,021 \\
\hline Coste total en Atención Primaria & $1.202,13$ & $1.179,53-1.225,46$ & $1.183,55$ & $1.161,21-1.206,91$ & 0,032 \\
\hline
\end{tabular}

(1) Modelo de ANCOVA: contrastes linealmente independientes entre las medias marginales estimadas. Evaluación de las covariables: índice de Charlson=0,6 y edad=67,1 años. Corrección: Bonferroni. Factores fijos: buen control y género. IC: intervalos de confianza del $95 \%$. p: significación estadística.

Valores expresados en euros $(€)$. NS: no significativo.

\section{Figura 1}

Porcentaje de buen control de la hipertensión arterial según la ausencia/presencia de enfermedad cardiovascular (A) y la edad de los pacientes (B)

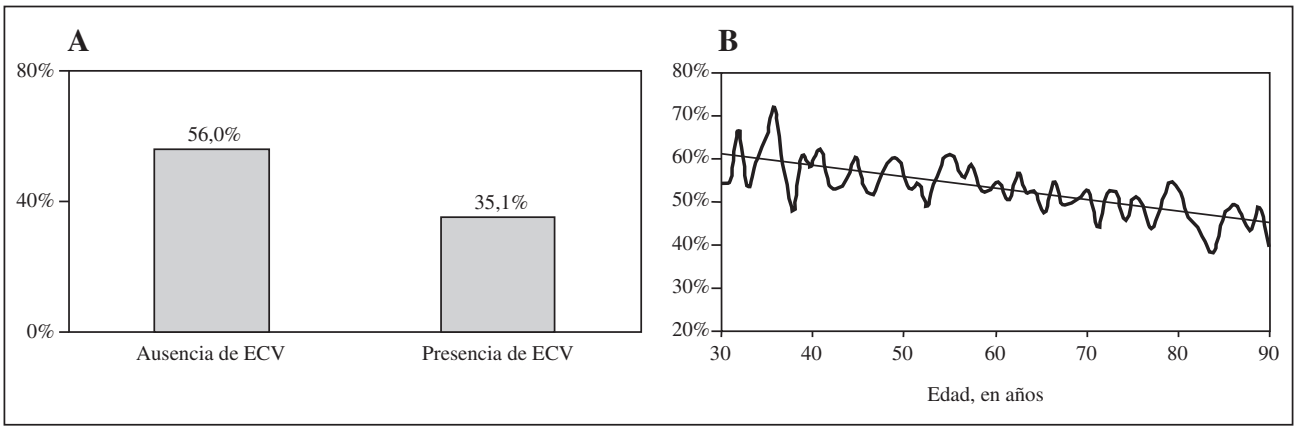

ECV: Enfermedad cardiovascular.

control fue del 52,0\% (n=7.091; IC: $51,2-$ $52,8 \%$ ), ver tabla 1 . Los pacientes según mal/buen control mostraron peor nivel de riesgo $(18,6 \pm 10,1$ vs. $14,5 \pm 9,3)$ y triglicéridos séricos $(140,6 \pm 88,0$ vs. $129,8 \pm 84,6$ $\mathrm{mg} / \mathrm{dL}$ ), $\mathrm{p}<0,001$ (tabla 2). En el modelo logístico, el mal control tuvo una relación independiente con la diabetes $(\mathrm{OR}=3,8)$, el ECV $(\mathrm{OR}=2,2)$ y los varones $(\mathrm{OR}=1,2)$, $\mathrm{p}<0,001$. El promedio/unitario del coste bruto fue de $1.278,22$ vs. $1.130,65 €$ $(\mathrm{p}<0,001) ; \mathrm{y}$ corregido de $1.202,13$ vs. $1.183,55 €(p=0,032)$, respectivamente. Estas diferencias se mantuvieron en todos los componentes del coste analizados. En la tabla 3 se detalla el modelo de costes directos corregidos según la presencia del control arterial. En la figura 1 se muestra la distribución del porcentaje de los pacientes en buen control de la hipertensión arterial, según la ausencia/presencia de enfermedad cardiovascular (A) y la edad (B).

\section{DISCUSIÓN}

La principal aportación de nuestro estudio sugiere que los pacientes en situación de mal control de la presión arterial presentan una mayor morbilidad cardiovascular con un ligero aumento en los costes directos. La organización de la AP de nuestro país, a través de la creciente informatización de sus centros, ofrece un marco idóneo para realizar este tipo de estudios retrospectivos de alcance poblacional en situación de práctica clínica habitual. El estudio incluye 
una amplia serie numérica de personas hipertensas $\quad(n=13.636$; prevalencia: $23,9 \%$ ), aspecto que puede realzar la consistencia de los resultados. Aunque cabe destacar que sin una adecuada estandarización de las metodologías, los resultados obtenidos de comorbilidad y costes deben de interpretarse con prudencia, obligándonos a ser cautelosos en la validez externa de los resultados ${ }^{16}$.

En función de los resultados del estudio, es posible deducir que en los próximos años se seguirán desarrollando ECV en la población, a pesar de seguir mejorando el control de la HTA $(52,0 \%)$, aspecto concordante con las recomendaciones de algunos grupos de expertos ${ }^{1-3,8,11,17-18}$. En este aspecto, es importante considerar que la HTA coexiste con otros factores de riesgo cardiovascular, sobre todo metabólicos (dislipemia, diabetes, obesidad [ver tabla 1]), para componer el perfil de riesgo cardiovascular individual ${ }^{1,11,18}$. Desde el punto de vista de la salud pública, parece razonable reforzar la prevención primaria, y la reducción de riesgos debe ser la mejor estrategia coste-efectiva a desarrollar. Por tanto, es primordial equilibrar las actividades de prevención con las de la atención a los sujetos que ya presentan enfermedad, y haciendo un mayor esfuerzo en mejorar los hábitos alimentarios de la población, en facilitar y fomentar la actividad física, y controlar el consumo de tabaco ${ }^{1-3,6-8,11,17-18}$.

En los pacientes diabéticos se encontró un deficiente control, concordante con otros estudios realizados $2-3,5-6,11,18-19$. En este colectivo de pacientes el objetivo a alcanzar por la estrategia terapéutica es también inferior al de la población general hipertensa y, por tanto, más difícil de conseguir. No sólo porque las cifras sean más bajas, sino porque también tenemos que considerar la lesión estructural del vaso arterial: remodelado hipertrófico, rigidez y mayor lesión endotelial ${ }^{19-20}$. Este aspecto puede ser de interés, puesto que el mayor coste se mues- tra en los pacientes con un mal control de la presión arterial (mayoritariamente diabéticos y con presencia ECV). Además, en el estudio se muestra que el mal control tuvo una asociación independiente con los varones y la presencia de ECV. Es posible que en los varones actúen factores de tipo genético, pero no se puede descartar que el cumplimiento terapéutico y el cambio de los hábitos de estilo de vida, tengan un peor seguimiento en este colectivo de pacientes $^{2,11,18}$. En cuanto a la presencia de ECV, seguramente se relacionen varios elementos multifactoriales con independencia de la diabetes $^{6,19}$. Cabe destacar, que las cifras de buen control de la tensión arterial suponen más dificultad de consecución $(<130 / 80$ $\mathrm{mmHg}$ ) para estos pacientes; pero además, en general se trata de pacientes polimedicados y con mayor carga de morbilidad, y es posible que en la práctica clínica, se establezcan niveles de prioridad en la consecución de los objetivos terapéuticos de control, donde los lípidos tengan un papel más relevante, que bajar las cifran de tensión arterial por debajo de las recomendadas de las guías de práctica clínica (aspecto consistente con nuestros resultados); aunque es indudable, que puedan existir otros factores de difícil cuantificación ${ }^{6,19-20}$. La asociación de la diabetes con el ECV repercute en una mayor dificultad en conseguir el descenso de la presión arterial, tal como han demostrado diferentes estudios de prevención de mortalidad y morbilidad ${ }^{2,6,11,19}$. Nuestros resultados nos hacen sentir optimistas, puesto que la mitad de los pacientes con HTA están adecuadamente controlados; no obstante hay que tener en cuenta que es una serie de casos reciente (año 2.006) y que aún nos queda un importante margen de mejora $^{1-6,18,21}$.

El promedio/unitario corregido (modelo ANCOVA) del coste total en hipertensos fue ligeramente superior en los sujetos que presentaron mal control de la HTA $(1.202,13$ frente a $1.183,55 € ; \mathrm{p}=0,032)$. Aunque estas diferencias son estadística- 
mente significativas, no son relevantes desde una perspectiva de eficiencia en la gestión clínica de los equipos de AP. En la bibliografía consultada existen pocas evidencias disponibles sobre el coste de la HTA en situación de práctica clínica, por tanto, nuestros resultados son de difícil comparación, pero posiblemente puedan ser de utilidad en la gestión de los recursos en centros reformados y urbanos similares a los nuestros ${ }^{9-10}$. Los estudios disponibles se sitúan en la vertiente de la evaluación fármaco-económica simulada, existiendo pocos estudios sobre el coste real de la enfermedad $^{9-10,22-23}$. En España, las cifras que manejaba el Ministerio de Sanidad y Consumo en 1994 acerca de los costes de la HTA, se situaron entre 1.200 y 1.600 millones de euros. Según Badía y colaboradores $^{9}$, los costes asistenciales se aproximaban al 30\% del total de los costes de la HTA y el $54 \%$ de los costes directos se producen en AP. Además, aunque es conocido, que el tratamiento farmacológico de la HTA es coste-efectivo, la sustitución de un medicamento antihipertensivo por otro en los pacientes no controlados, que constituye la práctica más frecuente en la actualidad, resulta a corto y medio plazo más caro y menos efectivo que la asociación de un segundo fármaco sinérgico al tratamiento habitual $^{11,24}$. Por tanto, planificar estrategias coste-efectividad, que consigan el control de presión sin alterar el metabolismo hidrocarbonato y sin acelerar la aparición de diabetes clínica, particularmente en la población hipertensa más joven, deberá redundar en una mayor prevención de morbilidad y mortalidad a largo plazo, que es la expectativa real de los pacientes de edad media que atendemos en nuestras consul$\operatorname{tas}^{24-25}$.

Algunas limitaciones del trabajo obligan a ser cautelosos en la generalización de los resultados. Entre ellas destaca el propio diseño del estudio, el diseño de costes (no se contabilizaron los de la atención especializada) o la posible variabilidad de los cen- tros en la coordinación con otros niveles asistenciales (continuum asistencial). El éxito en la atención de estos pacientes debería basarse en intervenciones de equipos pluridisciplinarios, que promuevan estrategias en las que los pacientes estén altamente comprometidos en su auto-cuidado. En general, es necesario tener mayor información sobre el grado de control de la HTA y de los costes asociados en condiciones reales de práctica clínica, lo que incluye considerar prioritariamente a la población de alto riesgo de padecer un $\mathrm{ECV}^{11}$. En conclusión, los pacientes en situación de mal control de la presión arterial presentan un similar consumo de recursos y costes. Debemos prestar más atención a la prevención primaria de los pacientes hipertensos, donde los profesionales sanitarios deberían de establecer medidas coste-efectivas encaminadas a fomentar estrategias de intervención en este colectivo de pacientes.

\section{AGRADECIMIENTOS}

A los profesionales sanitarios que con su constante introducción de datos día a día, hicieron posible la realización de este estudio.

\section{BIBLIOGRAFÍA}

1. Banegas JR. Epidemiología de la hipertensión arterial en España. Situación actual y perspectivas. Hipertensión. 2005; 22:353-62.

2. Coca A. Evolución del control de la hipertensión arterial en Atención Primaria en España. Resultados del estudio CONTROLPRES 2003. Hipertensión. 2005; 22:5-14.

3. Álvarez-Sala LA, Suárez C, Mantilla T, Franch J, Ruilope LM, Banegas JR, et al. Estudio PREVENCAT: control del riesgo cardiovascular en Atención Primaria. Med Clin (Barc). 2005; 124:406-10.

4. Gómez-Marcos MA, García-Ortiz L, GonzálezElena LJ, Ramos-Delgado E, González-García AM, Parra-Sánchez J. Efectividad de una inter- 
vención de mejora de calidad en el control de la presión arterial en Atención Primaria. Rev Clin Esp. 2006; 206:428-34.

5. Llisterri JL, Rodríguez GC, Alonso FJ, Lou S, Divisón JA, Santos JA, et al. Control de la presión arterial en la población hipertensa española atendida en atención primaria. Estudio PRESCAP 2002. Med Clin (Barc). 2004; 122:165-71.

6. García O, Lozano JV, Vegazo O, Jiménez FJ, Llisterri JL, Redón J. Control de la presión arterial de los pacientes diabéticos en el ámbito de atención primaria. Estudio DIAPA. Med Clin (Barc). 2003; 120:529-34.

7. Brotons C. Control de los factores de riesgo cardiovascular en Atención Primaria. ¿Controlamos los factores o controlamos el riesgo? Med Clin (Barc). 2005; 124:415-6.

8. Roca-Cusachs A. Control de la hipertensión arterial: la asignatura pendiente. Hipertensión. 2007; 24:1-3.

9. Badía X, Rovira J, Tresserras R, Trinxet C, Segú JL, Pardell H. El coste de la hipertensión arterial en España. Med Clin (Barc). 1992; 99:769-73.

10. Fender P, Guilhot J, Tilly B, Salanave B, Allemand $\mathrm{H}$. Treatment of severe arterial hypertension: cost of drug prescriptions in accordance with ANAES guidelines. Therapie. 2001; 56:111-8.

11. Grupo de Trabajo para el Tratamiento de la Hipertensión Arterial de la Sociedad Europea de Hipertensión y de la Sociedad Europea de Cardiología. ESH/ESC 2007 Guidelines for the management of arterial hypertension. Rev Esp Cardiol. 2007; 60: 968.e1-94.

12. Lamberts H, Wood M, Hofmans-Okkes IM (eds). The International Classification of Primary Care in the European Community. With a multi-language layer. Oxford: Oxford University Press, 1993.

13. Weiner JP, Starfield BH, Steinwachs DM, Mumford LM. Development and application of a population-oriented measure of ambulatory care casemix. Med Care. 1991: 29:452-72.

14. Sicras-Mainar A, Serrat-Tarrés R, Navarro-Artieda R, Llopart-López JR. Posibilidades de los Grupos Clínicos Ajustados (Ajusted Clinical GroupsACGs) en el ajuste de riesgos de pago capitativo. Rev Esp Salud Pública 2006; 80:55-65.
15. Thompson SG, Barber JA. How should cost data in pragmatic randomised trials be analysed? BMJ. 2000; 320:1197-200.

16. Sackett D, Rosenberg W, Gray J, Haynes Rb. Richardson WS. Evidence based medicine: what it is and what it isn't. BMJ. 1996; 312:71-72.

17. Coca A. Grado de control de la hipertensión arterial en España: ¿dónde estamos? Hipertensión 2004; $21: 385-7$

18. Banegas JR, Graciani A. Hipertensión arterial. Objetivos terapéuticos, magnitud y manejo. Cardiovasc Risk Factors. 2005; 14:150-9.

19. Gaede P, Vedel P, Larsen N, Jensen G, Parving $\mathrm{HH}$, Pedersen O. Multifactorial intervention and cardiovascular disease in patients with type 2 diabetes. N Engl J Med 2003; 348:383-93.

20. Emberson JR, Whincup PH, Morris RW, Walker $\mathrm{M}$. Re-assessing the contribution of serum total cholesterol, blood pressure and cigarette smoking to the aetiology of coronary heart disease: impact of regression dilution bias. Eur Heart J. 2003; 24:1719-26.

21. Benítez M, Pérez S, Dalfó A, Piqueras MM, Losada G, Vila MA. Estudio DISEHTAC II: diagnóstico y seguimiento de la hipertensión arterial en Cataluña. Comparación con los datos de 1996. Aten Primaria. 2005; 35:7-11.

22. Bonet A, Gosalbes V, Fito M, Navarro J. Rational prescribing and cost reduction in the treatment of arterial hypertension: a simulation exercise. Gac Sanit. 2001; 15:327-335.

23. Antoñanzas F, Velasco M, Abbas I, Pontes C, Delgadillo J, Terán M. Modelo teórico de análisis coste-efectividad de la terapia combinada de enalapril-nitrendipino en el tratamiento de la hipertensión arterial. Aten Primaria 2003; 31:366-71.

24. The State of Health Care Quality 2004. Washington, DC: National Committee for Quality Assurance; 2004.

25. Weber M, Julius S, Kjeldsen S, Brunner HR, Ekman S, Hansson L, et al. Blood pressure dependent and independent effects of antihypertensive treatment on clinical events in the VALUE trial. Lancet. 2004; 363:2049-51. 